\title{
Relationship between cerebral blood flow and later cognitive decline in hypertensive patients with cerebral small vessel disease
}

\author{
Kazuo Kitagawa ${ }^{1}$, Naohiko $\mathrm{Oku}^{2}$, Yasuyuku Kimura ${ }^{3}$, Yoshiki Yagita ${ }^{1}$, Manabu Sakaguchi ${ }^{1}$, Jun Hatazawa ${ }^{3}$ \\ and Saburo Sakoda ${ }^{1}$
}

Vascular risk factors are thought to be important for dementia. However, there is little evidence for a prospective association between cerebral blood flow and the risk of cognitive decline. Twenty-seven cognitively intact hypertensive patients aged 55 years and older with lacunar infarction or white matter lesions in magnetic resonance imaging (MRI) underwent positron emission tomography (PET) to measure cerebral blood flow (CBF) and cerebral vascular reactivity (CVR). Cognitive function was assessed at baseline and 3 years later with the mini-mental state examination (MMSE). Patients whose MMSE score fell by more than three points were classified as having cognitive decline. Six patients showed cognitive decline. Baseline CBF in these patients was significantly lower than that of the 21 patients without cognitive decline $(31.2 \pm 2.4 \mathrm{vs} .42 .6 \pm 5.9 \mathrm{ml}$ per $100 \mathrm{~g} \mathrm{~min}^{-1}$, respectively; $P<0.001$ ). A moderate linear association was found between CBF and change in MMSE score over a 3-year period $(r=0.59, P=0.001)$, not between CBF and baseline MMSE score. In contrast, no association between CVR and later cognitive decline was found. This study suggests that cerebral hypoperfusion is associated with later cognitive decline. Hypertension Research (2009) 32, 816-820; doi:10.1038/hr.2009.100; published online 3 July 2009

Keywords: cerebral blood flow; cerebral small vessel disease; cognitive decline; positron emission tomography

\section{INTRODUCTION}

Observational epidemiological studies have shown that midlife vascular risk factors, particularly hypertension, and the level of atherosclerosis are closely associated with dementia later in life. ${ }^{1,2}$ Hypertension alters the structure of cerebral blood vessels by inducing hypertrophy, remodeling and stiffening through oxidative stress and endothelial dysfunction, which result in a slight reduction in cerebral blood flow (CBF), attenuation of the brain activation-induced increase in $\mathrm{CBF}$ and impaired cerebral autoregulation. ${ }^{3}$ Advancing age and vascular risk factors undoubtedly accelerate not only atherosclerosis in the circle of Willis but also lipohyalinosis in the cerebral small vessels, possibly causing chronic cerebral hypoperfusion. The vascular hypothesis proposed by de la Torre ${ }^{4}$ states that chronic brain hypoperfusion is a critical factor for Alzheimer's disease. However, there is little evidence for a prospective association between cerebral blood flow and the risk of cognitive decline and dementia. Several cross-sectional positron emission tomography (PET) studies have shown that cerebral hypoperfusion is generally associated with reduced cerebral metabolism in dementia. ${ }^{5,6}$ To date, only one prospective study has provided evidence linking cerebral hypoperfusion to the pathogenesis of dementia. Using transcranial Doppler ultrasonography, Ruitenberg et al. ${ }^{7}$ showed that reduced flow velocity in the middle cerebral artery is associated with dementia and cognitive decline in a prospective 6-year follow-up study. Transcranial Doppler ultrasonography is a noninvasive, inexpensive method of measuring cerebral hemodynamics, but an absolute value of CBF is rarely calculated. In contrast, PET is the gold standard for measuring $\mathrm{CBF}^{8,9}$ Recent epidemiological studies have found that the presence of cerebral small vessel diseases, such as silent brain infarctions ${ }^{10}$ and white matter lesions, ${ }^{11}$ independently predict later dementia and cognitive decline. Thus, we enrolled hypertensive patients with lacunar infarctions or white matter lesions in their brain MRI, presumptive high-risk patients for cognitive decline in this study. The purpose of this study is to clarify the associations between blood flow and cognitive decline in hypertensive patients with cerebral small vessel disease using PET.

\section{METHODS}

\section{Patients}

Twenty-seven cognitively intact patients with essential hypertension accompanied by evidence of ischemic white matter lesions by brain MRI were enrolled from among outpatients of Osaka University Hospital. The protocol was approved by the institutional review board and ethics committee of Osaka University Medical School. Patients were excluded from the study if they had 
experienced a clinical cerebrovascular event in the previous 3 months, if they showed severe stenosis $(\geqq 70 \%)$ of the carotid artery and/or middle cerebral artery, or if they presented with any type of dementia.

\section{MRI}

All MRI was performed with a 1.5-T. Signa Horizon (GE Yokogawa Medical Systems Ltd, Tokyo, Japan) scanner. The imaging protocol consisted of a T2weighted spin-echo, T1-weighted spin-echo and fluid-attenuated inversionrecovery (FLAIR) imaging. Lacunar infarct was defined as an area of focal hyperintensity $\geqq 3 \mathrm{~mm}$ in diameter on T2-weighted images with corresponding low signal intensity on T1-weighted images. To exclude the dilated perivascular space, a diagnosis of lacunar infarct also required that the lesion be surrounded by a hyperintense gliotic rim on FLAIR images. White matter lesions were rated visually on axial FLAIR images using the Schelten rating scale with modifications (range 0 to 30), ${ }^{12}$ in which scores 0 to 6 were given for four subcortical regions (frontal, parietal, occipital, temporal) and scores of 0 to 2 were given for three periventricular regions (frontal caps, occipital caps, lateral bands). The severity of brain subcortical atrophy was estimated by calculating the ratio of ventricle to brain on T1-weighted images as an Evans index. Cortical atrophy was assessed using the rating scales for sulcal dilatation (frontal, parietal, temporal, occipital, sylvian fissure) as described. ${ }^{13}$ A subscore of 0 (absent), 1 (mild), 2 (moderate) or 3 (severe) was given for each region to qualify cortical atrophy. The cortical atrophy score was the sum of the four subscores (range: $0-15$ ).

\section{PET}

To measure $\mathrm{CBF}$, we used PET with an ${ }^{15} \mathrm{O}$-labeled water injection as described. ${ }^{8,9}$ We used a high-performance PET scanner (SET-2400W; Shimadzu Co., Kyoto, Japan) that uses 63 slices (slice thickness of $3.1 \mathrm{~mm}$ ) and a spatial resolution of $3.7 \mathrm{~mm}$ full width at half maximum. Regional CBF was quantitatively measured using PET, an ${ }^{15} \mathrm{O}$-labeled water injection, and an autoradiographic method. Then, to assess the capacity of cerebral vascular reserve, two additional scans were performed in vasodilated condition after an intravenous injection of acetazolamide titrated to $1000 \mathrm{mg}$. At the end of every scan, the patient's blood pressure, pulse rate and arterial blood gas tensions were measured. The averaged data of two measurements were obtained for both baseline and vasodilated conditions.

Regional CBF PET image data sets were realigned and transformed stereotaxically into an identical normal brain template with statistical parametric mapping software (SPM99; Wellcome Department of Cognitive Neurology, University College London, London, UK) running on MATLAB 5.3 (The MathWorks Inc., Natic, MA, USA) for Windows (Microsoft Inc., Redmond, WA, USA). Regional cerebrovascular reserve (CVR) images were calculated with the realigned sets of CBF images. Identical regions of interest (the whole cerebrum, frontal cortex, temporal cortex, occipital cortex, parietal cortex, basal ganglia areas, thalami) were drawn on standardized CBF images. The region of interest for the whole cerebrum was drawn manually on all slices of the standardized MRI T1 template provided by SPM99 and applied to the standardized CBF images. Each of the remaining regions of interest consisted of multiple small circular regions of interest $(16 \mathrm{~mm}$ in diameter) linked together.

\section{Assessment of cognitive function and dementia}

Cognitive function was assessed for all patients at baseline using the minimental state examination (MMSE). ${ }^{14}$ Scores range from 0 to 30 across seven cognitive domains: orientation to time ( 5 points), orientation to place (5 points), registration of three words (3 points), attention and calculation ( 5 points), recall of 3 words ( 3 points), language ( 8 points) and visual construction (1 point), with a lower score indicative of a greater degree of cognitive impairment. A score of 24 points or lower indicates some degree of cognitive impairment. ${ }^{15}$ During the follow-up period, the MMSE was repeated if the patient or their family complained of progressive memory impairment. Otherwise, the MMSE was repeated after 3 years. If the MMSE score fell by more than three points in the 3-year period, the patient was classified with cognitive decline according to the criteria in PROGRESS trial. ${ }^{16}$ Patients with possible dementia incidents (MMSE score $<24$ or declined $\geqq 3$ points in
3 years) were assessed for dementia by a neurologist in accordance with the Diagnostic Statistical Manual of Mental Disorders, edition III, revised (DSMIIIR). ${ }^{17}$ Patients who were classified with dementia were also diagnosed with either Alzheimer's disease or vascular dementia according to the National Institute of Neurological and Communication Disorders-Alzheimer's Disease and Related Disorders Association (NINCDS-ADRDA), ${ }^{18}$ and the National Institute of Neurological Disorders and Stroke-Association Internationale pour la Recherche et I'Enseignement en Neurosciences (NINDS-AIREN). ${ }^{19}$ Three neurologists who were blinded to the clinical information assessed each case.

\section{Measurement of cardiovascular risk factors and carotid atherosclerosis}

Blood pressure was measured at the right brachial artery using a sphygmomanometer in the sitting position. Fasting blood glucose, serum total cholesterol, high-density lipoprotein cholesterol, triglycerides and creatinine levels were determined from the blood sample. Information on patient education, medical history and medication use was obtained by interviewing the patient and from clinical records. Diabetes mellitus was defined as fasting blood glucose levels $\geqq 126 \mathrm{mg}$ per $100 \mathrm{ml}$, HbAlc $\geqq 5.8 \%$ or by the use of glucoselowering agents. Dyslipidemia was defined as fasting serum total cholesterol $\geqq 220 \mathrm{mg}$ per $100 \mathrm{ml}$, triglyceride $\geqq 150 \mathrm{mg}$ per $100 \mathrm{ml}$, high-density lipoprotein cholesterol $<40 \mathrm{mg}$ per $100 \mathrm{ml}$ or the use of cholesterol-lowering agents. Smoking status was categorically evaluated from self-reports; a smoker was defined as a person currently smoking $\geqq 10$ cigarettes per day for $\geqq 1$ year.

The severity of carotid atherosclerosis was evaluated with duplex carotid ultrasonography as described. ${ }^{20}$ All ultrasound examinations were performed with a Phillips SONOS 5500 (Phillips Medical Systems, Tokyo, Japan) equipped with a $7.5-\mathrm{MHz}$ linear-array transducer. The intima-media thickness, defined as the distance between the intima-luminal interface and the media-adventitial interface, was measured as described. We calculated the mean carotid intimamedia thickness (mean intima-media thickness) by averaging the thickness at 12 sites: the near and far walls of both the right and left distal common carotid artery, carotid bifurcation and internal carotid artery.

\section{Data analysis}

Statistical analyses were performed with SPSS 9.0J. for Windows (SPSS Japan Inc., Tokyo, Japan). To analyze the relation between cognitive decline and patient characteristics, we used a $\chi^{2}$-test for categorical data and unpaired $t$-test for continuous data. The effects of acetazolamide on increased CBF were analyzed using a one-way analysis of variance. Linear regression analysis was used to examine the association between CBF and baseline MMSE or change in MMSE score over a period of 3 years. When not otherwise specified, data are presented as means \pm s.d. A two-tailed $P$-value $<0.05$ was considered statistically significant.

\section{RESULTS}

Table 1 shows the patients' baseline characteristics. The mean baseline MMSE score was 27.5 points. No patients developed stroke or cardiovascular events during the follow-up period. Among 27 patients, the change in the MMSE score over the 3-year period was $-0.96 \pm 2.58$ points. Six patients experienced cognitive decline with a drop of 3 points or more during the 3 -year follow-up period. Of those, three were diagnosed with dementia (two with Alzheimer's disease and one with vascular dementia). Univariate analysis revealed that cognitive decline was more prevalent in men. However, age, education, blood pressure, serum lipid and creatinine levels, fasting glucose levels, smoking, alcohol consumption and stroke history at baseline were similar in patients with and without later cognitive decline. Baseline MMSE scores, severity of carotid intima-media thickness, white matter lesions, cortical atrophy, subcortical atrophy and the prevalence of lacunes were also similar in the two groups. The frequency of angiotensin-converting enzyme inhibitor/angiotensin II receptor blocker, calcium channel blocker, statin and aspirin use was also similar in the two groups. The baseline CBF in patients with later 
Table 1 Characteristics of the study patients at baseline

\begin{tabular}{|c|c|c|c|c|}
\hline & \multirow[b]{2}{*}{ Total } & \multicolumn{2}{|c|}{ Later cognitive decline } & \multirow[b]{2}{*}{ P-value } \\
\hline & & $(-)$ & $(+)$ & \\
\hline Age, years & $64.8 \pm 7.5$ & $64.5 \pm 7.8$ & $65.8 \pm 6.6$ & 0.71 \\
\hline Gender, men, \% & 55.6 & 42.9 & 100 & 0.02 \\
\hline Education, years & $12(9-21)$ & $12(9-21)$ & $12(11-16)$ & 0.24 \\
\hline \multicolumn{5}{|l|}{ Blood pressure, $\mathrm{mm} \mathrm{Hg}$} \\
\hline Systolic & $135.8 \pm 11.0$ & $135.9 \pm 10.5$ & $135.3 \pm 13.8$ & 0.91 \\
\hline Diastolic & $81.9 \pm 7.4$ & $80.2 \pm 7.1$ & $87.7 \pm 5.4$ & 0.03 \\
\hline Use of ACEI/ARB, \% & 70.4 & 66.7 & 83.3 & 0.63 \\
\hline Use of $\mathrm{CCB}, \%$ & 66.7 & 71.4 & 50.0 & 0.37 \\
\hline Statin use, $\%$ & 37.0 & 42.9 & 16.7 & 0.36 \\
\hline Diabetes mellitus, \% & 14.8 & 14.3 & 16.7 & 1.00 \\
\hline Fasting blood glucose (mg per $100 \mathrm{ml}$ ) & $104.4 \pm 17.5$ & $104.7 \pm 18.0$ & $103.5 \pm 17.5$ & 0.89 \\
\hline $\mathrm{HbA} 1 \mathrm{C}, \%$ & $5.6 \pm 0.8$ & $5.5 \pm 0.8$ & $5.7 \pm 0.7$ & 0.73 \\
\hline Creatinine, mg per $100 \mathrm{ml}$ & $0.79 \pm 0.16$ & $0.78 \pm 0.16$ & $0.80 \pm 0.14$ & 0.80 \\
\hline Smoking, \% & 51.9 & 42.9 & 83.3 & 0.17 \\
\hline Alcohol consumption, \% & 29.6 & 23.8 & 50.0 & 0.32 \\
\hline History of stroke & 18.5 & 19.0 & 16.7 & 1.00 \\
\hline Aspirin use, \% & 22.2 & 19.0 & 33.3 & 0.59 \\
\hline \multicolumn{5}{|l|}{$M R I$} \\
\hline CVR (\%) & $51.4 \pm 21.8$ & $51.5 \pm 23.8$ & $51.3 \pm 16.9$ & 0.80 \\
\hline
\end{tabular}

Abbreviations: ACEI, angiotensin-converting enzyme inhibitor; ARB, angiotensin II type 1 receptor blocker; CCB, calcium channel blocker; CVR, cerebral vascular reactivity; IMT, intima-media thickness; MMSE, mini-mental state examination.

Values are unadjusted mean \pm s.d., median (range) or percentage. Cognitive decline $(-)$ and $(+)$ indicate the absence and presence of later cognitive decline during the 3 -year follow-up period, respectively.

cognitive decline was significantly lower than that in the 21 patients without later cognitive decline $(31.2 \pm 2.4$ vs. $42.6 \pm 5.9 \mathrm{ml}$ per $100 \mathrm{~g} \mathrm{~min}^{-1}$, respectively; $P<0.001$ ) (Figure 1 ). The same relationships were observed in every brain region. Local CBF values in patients with cognitive decline were significantly lower than those in patients without in the frontal cortex $(33.9 \pm 3.7$ vs. $45.3 \pm 6.1 \mathrm{ml}$ per $\left.100 \mathrm{~g} \mathrm{~min}^{-1}, P<0.01\right)$, temporal cortex $(33.3 \pm 4.1$ vs. $48.5 \pm 7.9 \mathrm{ml}$ per $\left.100 \mathrm{~g} \mathrm{~min}^{-1}, P<0.01\right)$, occipital cortex $(38.7 \pm 5.6 v s .55 .0 \pm 8.0 \mathrm{ml}$ per $\left.100 \mathrm{~g} \mathrm{~min}^{-1}, P<0.01\right)$, parietal cortex $(33.1 \pm 3.6$ vs. $47.9 \pm 6.4 \mathrm{ml}$ per $\left.100 \mathrm{~g} \mathrm{~min}^{-1}, \quad P<0.01\right)$, basal ganglia areas $(39.5 \pm 5.6 \mathrm{vs}$. $52.5 \pm 9.6 \mathrm{ml}$ per $\left.100 \mathrm{~g} \mathrm{~min}^{-1}, P<0.01\right)$ and thalami $(40.3 \pm 5.7 \mathrm{vs}$. $51.9 \pm 10.7 \mathrm{ml}$ per $100 \mathrm{~g} \mathrm{~min}^{-1}, P<0.02$ ). A moderate linear association was found between $\mathrm{CBF}$ and change in the MMSE score during the 3 -year period $(r=0.59, P=0.001)$, although no correlation was found between CBF and the baseline MMSE score $(r=0.13, P=0.51)$.
In contrast, the cerebrovascular reserve after acetazolamide administration did not differ between patients with and without cognitive decline ( $51.4 \pm 16.9$ vs. $51.5 \pm 23.8 \%$, respectively, $P=0.80$ ). Representative PET scans of patients with and without later cognitive decline are shown in Figure 2. Although both cases had similar levels of white matter lesions and cerebral atrophy, the patient with lower CBF developed cognitive decline and dementia, whereas cognitive function remained intact in the patient with higher CBF.

\section{DISCUSSION}

Using PET, we found that lower baseline CBF levels are related to a higher prevalence of cognitive decline over a 3-year follow-up period. Epidemiological studies have shown that most of Alzheimer's disease risk factors are vascular related. Among the vascular risk factors, hypertension, particularly during mid-life, is a powerful trigger for 


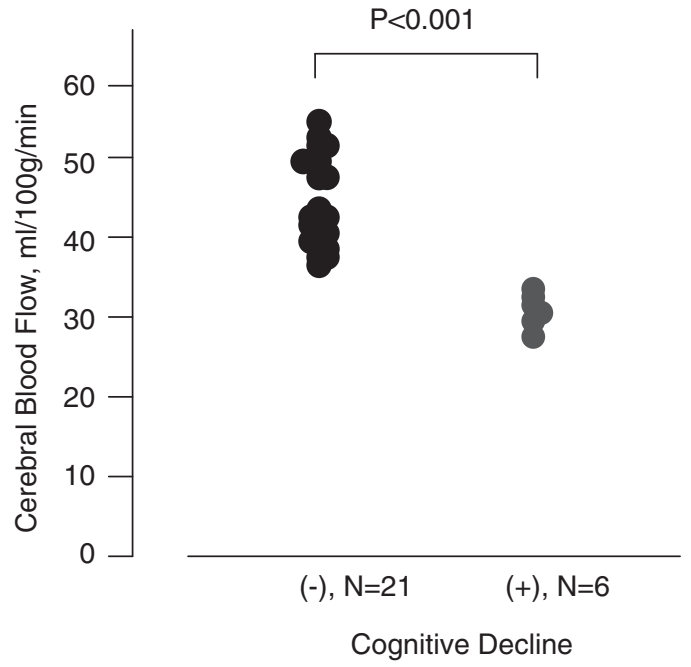

Figure 1 Scatter diagram of the cerebral blood flow (CBF) values and the incidence of cognitive decline. The CBF values of patients who later experienced cognitive decline were significantly lower than those without later cognitive decline $(P<0.001)$.

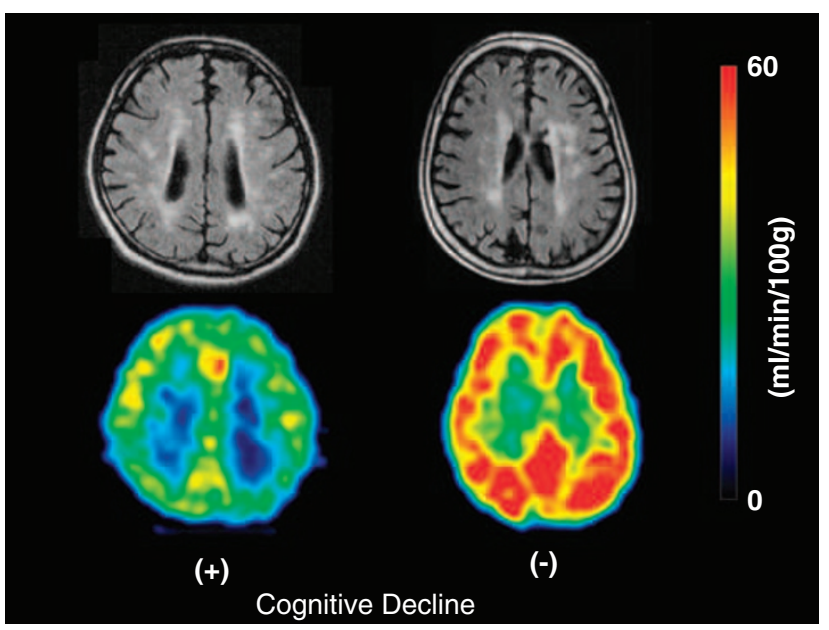

Figure 2 Representative positron emission tomography (PET) and magnetic resonance imaging (MRI) images of patients with and without later cognitive decline. Both patients had similar mini-mental state examination (scores (25 and 26), and the severity of white matter lesions and cerebral atrophy were similar as shown in MRI FLAIR images in the upper panels. However, the 71-year-old male patient with lower cerebral blood flow (CBF) values $\left(30.6 \mathrm{ml} \mathrm{min}^{-1}\right.$ per $\left.100 \mathrm{~g}\right)$ shown on the left developed cognitive decline and dementia, whereas cognitive function remained intact in the 82-year-old female patient with higher CBF values $\left(47.1 \mathrm{ml} \mathrm{min}^{-1}\right.$ per $100 \mathrm{~g}$ ) shown on the right.

dementia and cognitive decline in the elderly. ${ }^{21,22}$ Some randomized controlled trials also show a reduced risk of dementia with antihypertensive drug treatment. ${ }^{23-25}$ Sustained systemic hypertension triggers small vessel atherosclerosis of the intracranial vasculature, possibly leading to lacunar infarctions and white matter lesions. These small vessel diseases are associated with cognitive function in the elderly. ${ }^{26,27}$ Although the amyloid hypothesis of Alzheimer's disease is more generally accepted, ${ }^{28}$ systemic atherosclerosis ${ }^{2,29}$ and cerebral small vessel atherosclerosis ${ }^{8,9}$ have been identified as independent predictors for later cognitive decline and dementia in apparently healthy individuals. Although cerebral hypoperfusion is regarded as a crucial factor in vascular hypothesis, ${ }^{30}$ there is little evidence for a prospective association between cerebral hemodynamics and risk of cognitive decline. Reduced flow velocity in the middle cerebral artery preceded dementia in a large population-based study. ${ }^{7}$ However, the flow velocity value may be affected by a number of factors independent of the blood flow itself, including the insonation angle between the ultrasound beam and the middle cerebral artery, the size of vessel lumen, arterial stiffness and the presence of aortic atherosclerosis and/or aortic valve disease. Neuroimaging techniques, such as PET and single photon emission computed tomography, are the gold standard for measuring CBF and metabolism. One of the most consistent findings in Alzheimer's disease patients is a reduction of metabolism and perfusion in the posterior temporo-parietal, posterior cingulate and frontal regions. ${ }^{31}$ Longitudinal studies have also shown that a decline in glucose metabolism or perfusion in discrete brain areas, such as the temporal cortex, is predictive of future cognitive decline in patients with mild cognitive impairment ${ }^{32}$ or Alzheimer's disease. ${ }^{5,33}$ However, the involvement of vascular factors in Alzheimer's disease is supported by PET studies conducted by Nagata et al. ${ }^{34}$ In their study, the oxygen extraction fraction was increased in Alzheimer's disease patients, whereas both the CBF and oxygen metabolism were decreased relative to the control group, suggesting that cerebral hypoperfusion at the capillary level is involved in Alzheimer's disease. Herein, we tried to clarify the causal relation between $\mathrm{CBF}$ and cognitive decline in a prospective quantitative PET study.

Our findings suggest that cerebral hypoperfusion precedes cognitive decline. In some trials, cognitive ability improves when cerebral hypoperfusion in patients with severe carotid stenosis is reversed by carotid revascularization without complications, ${ }^{35}$ supporting the vascular hypothesis. In contrast to baseline CBF, baseline CVR is almost the same between patients with and without later cognitive decline. Although CVR after an acetazolamide challenge is a useful index for detecting misery perfusion in carotid occlusive patients, ${ }^{36}$ the significance of CVR in cerebral small vessel disease remains unclear. Although the severity of white matter lesions, brain atrophy and lacunes is similar between the two groups (Table 1), the findings that the patients with later cognitive decline had reduced CBF and preserved CVR may indicate that ischemia-related neuronal $\operatorname{loss}^{37}$ or cortical disconnections owing to subcortical lesions ${ }^{38}$ is involved with cortical hypoperfusion in these patients.

There are several limitations to our study. First, the number of patients in our study is only 27 . The small sample size did not allow us to perform multiple regression analyses by controlling for a large number of variables such as age, gender, education, MMSE score, stroke history, traditional risk factors and MRI findings. However, the difference in such variables is unlikely to contribute to lower CBF in patients with later cognitive decline because the frequency and values of those variables, except for gender, were almost similar between the two groups (Table 1). Second, our participants were hypertensive patients with evidence of cerebral small vessel disease who may be at higher risk for Alzheimer's disease. It remains unclear whether our findings can also be extrapolated to normal elderly patients without hypertension or any evidence of white matter lesions in MRI. Third, PET and MRI were only performed at baseline. Whether cognitive decline parallels decreased CBF and progression of cerebral small vessel disease during the follow-up period requires further examination. Lastly, the neuropsychological evaluation was based only on MMSE scores in this study. Other neuropsychological testing such as 
executive function, mental speed and motor control could be also useful for detecting any change of cognitive function in patients with cerebral small vessel disease.

In conclusion, our findings suggest that chronic cerebral hypoperfusion is associated with the development of cognitive decline. Aggressive therapy to preserve or improve CBF in hypertensive patients who show signs of white matter lesions might be considered as a measure to prevent the onset of cognitive decline.

\section{ACKNOWLEDGEMENTS}

This study was supported in part by the research grant for cardiovascular disease from the Japanese Ministry of Health, Welfare and Labor and by the Smoking Research Foundation of Japan. We are grateful to the cyclotron staff of Osaka University Hospital for technical support. We also thank Ms C Kurano and Ms M Nishiyama for secretarial assistance.

1 Kivipelto M, Ngandu T, Laatikainen T, Winblad B, Soininen H, Tuomilehto J. Risk factor for the prediction of dementia risk in 20 years among middle aged people: a longitudinal, population-based study. Lancet Neurol 2006; 5: 735-741.

2 van Oijen M, de Jong FJ, Witteman JCN, Hofman A, Koudstaal PJ, Breteler MM. Atherosclerosis and risk of dementia. Ann Neurol 2007; 61: 403-410.

3 ladecola C, Davisson RL. Hypertension and cerebrovascular dysfunction. Cell Metabolism 2008; 7: 476-484.

4 de la Torre JC. Is Alzheimer's disease a neurodegenerative or a vascular disorder? Data, dogma, and dialectics. Lancet Neurol 2004; 3: 184-190.

5 Silverman DHS, Small GW, Chang CY, Alexander GE, Schapiro MB, Jagust WJ, Hoffman JM, Welsh-Bohmer KA, Alavi A, Clark CM, Salmon E, de Leon MJ, Mielke R, Cummings $\mathrm{JL}$, Kowell AP, Gambhir SS, Hoh CK, Phelps ME. Positron emission tomography in evaluation of dementia. Regional brain metabolism and long-term outcome. JAMA 2001; 286: 2120-2127.

6 Dickerson BS, Sperling RA. Neuroimaging biomarkers for clinical trials of diseasemodifying therapies in Alzheimer's disease. NeuroRx 2005; 2: 348-360.

7 Ruitenberg A, den Heijer T, Bakker SL, van Swieten JC, Koudstaal PJ, Hofman A, Breteler MM. Cerebral hypoperfusion and clinical onset of dementia: The Rotterdam Study. Ann Neurol 2005; 57: 789-794.

80 oku N, Kitagawa K, Imaizumi M, Takasawa M, Piao R, Kimura Y, Kajimoto K, Matsumoto M, Hori M, Hatazawa J. Hemodynamic influences of losartan on the brain in hypertensive patients. Hypertens Res 2005; 28: 43-49.

9 Kimura Y, Kitagawa K, Oku N, Kajimoto K, Kato H, Tanaka M, Sakaguchi M, Hougaku $\mathrm{H}$, Sakoda S, Hatazawa J. Hemodynamic influences of azelnidipine on the brain in hypertensive patients. Hypertens Res 2008; 31: 2147-2154.

10 Vermeer SE, Longstreth Jr WT, Koudstaal PJ. Silent brain infarcts: a systematic review. Lancet Neurol 2007; 6: 611-619.

11 Schmidt R, Petrovic K, Ropele S, Enzinger C, Fazekas F. Progression of leukoaraiosis and cognition. Stroke 2007; 38: 2619-2625.

12 Scheltens P, Barkhof F, Leys D, Pruvo JP, Nauta JJP, Vermersch P, Steinling M, Valk J. A semiquantitative rating scale for the assessment of signal hyperintensities on magnetic resonance imaging. J Neurol Sci 1993; 114: 7-12.

13 Pasquier F, Leys D, Weerts JGE, Mounier-Vehier F, Barkhof F, Scheltens P. Inter- and intraobserver reproducibility of cerebral atrophy assessment on MRI scans with hemispheric infarcts. Eur Neurol 1996; 36: 268-272.

14 Folstein MF, Folstein SE, McHugh PR. 'Mini-mental state'. A practical method for grading the cognitive state of patients for the clinician. J Psychiatr Res 1975; 12: 189-198.

15 Helkala EL, Kivipelto M, Hallikainen M, Alhainen K, Heinonen H, Tuomilehto J, Soininen H, Nissinen A. Usefulness of repeated presentation of Mini-Mental State Examination as a diagnostic procedure-a population-based study. Acta Neurol Scand 2002; 106: 341-346.

16 Tzourio C, Anderson C, Chapman N, Woodward M, Neal B, MacMahon S, Chalmers J, PROGRESS Collaborative Group. Effects of blood pressure lowering with perindopril and indapamide therapy on dementia and cognitive decline in patients with cerebrovascular disease. Arch Intern Med 2003; 163: 1069-1075.

17 American Psychiatric Association. Diagnostic and Statistical manual of Mental Disorders. APA: Washington, DC, 1987

18 McKhann G, Drachman D, Folstein M, Katzman R, Price D, Stadlan EM. Clinical diagnosis of Alzheimer's disease: report of the NINCDS-ADRDA Work Group under the auspices of Department of Health and Human Service Task Force on Alzheimer's Disease. Neurology 1984; 34: 939-944.

19 Roman GC, Tatemichi TK, Erkinjuntti T, Cummings JL, Masdeu JC, Garcia JH, Amaducci L, Orgogozo JM, Brun A, Hofman A. Vascular dementia: diagnostic criteria for research studies. Report of the NINDS-AIREN International Workshop. Neurology 1993; 43: 250-260.

20 Kitagawa K, Hougaku H, Yamagami H, Hashimoto H, Itoh T, Shimizu Y, Takahashi D, Murata S, Seike Y, Kondo K, Hoshi T, Furukado S, Abe Y, Yagita Y, Sakaguchi M, Tagaya M, Etani H, Fukunaga R, Nagai Y, Matsumoto M, Hori M. Carotid intima-media thickness and risk of cardiovascular events in high-risk patients. Results of the Osaka Follow-Up Study for Carotid Atherosclerosis 2 (OSACA2 Study). CerebrovasC Dis 2007; 24: 35-42.

21 Skoog I, Lernfelt B, Landahl S, Palmertz B, Andreasson LA, Nilsson L, Persson G, Odén A, Svanborg A. A 15 year longitudinal study of blood pressure and dementia. Lancet 1996; 347: 1141-1145.

22 Qiu C, Winblad B, Fratiglioni L. The age-dependent relation of blood pressure to cognitive function and dementia. Lancet Neurol 2005; 4: 487-499.

23 Forette F, Seux ML, Staessen JA, Thijs L, Birkenhager WH, Babarskiene MR, Babeanu S, Bossini A, Gil-Extremera B, Girerd X, Laks T, Lilov E, Moisseyev V, Tuomilehto J, Vanhanen $\mathrm{H}$, Webster J, Yodfat $Y$, Fagard R. Prevention of dementia in randomized double-blind placebo-controlled systolic hypertension in Europe (Syst-Eur) trial. Lancet 1998; 352: 1347-1351.

24 Birns J, Morris R, Donaldson N, Kalra L. The effects of blood pressure reduction on cognitive function: a review of effects based on pooled data from clinical trials. J Hypertens 2006; 24: 1907-1914.

25 Peters R, Beckett N, Forette F, Tuomilehto J, Clarke R, Ritchie C, Waldman A, Walton I, Poulter R, Ma S, Comsa M, Burch L, Fletcher A, Bulpitt C, for the HYVET investigator. Incident dementia and blood pressure lowering in the hypertension in the very elderly trial cognitive function assessment (HYVET-COG): a double-blind, placebo controlled trial. Lancet Neurol 2008; 7: 683-689.

26 van der Flier WM, van Straaten ECW, Barkhof F, Verdelho A, Madureira S, Pantoni L, Inzitari D, Erkinjuntti T, Crisby M, Waldemar G, Schmidt R, Fazekas F, Scheltens P, on behalf of the LADIS Study Group. Small vessel disease and general cognitive function in nondisabled elderly. The LADIS Study. Stroke 2005; 36: 2116-2120.

27 Benisty S, Gouw AA, Porcher R, Madureira S, Hernandez K, Poggesi A, van der Flier WM, van Straaten EC, Verdelho A, Ferro J, Pantoni L, Inzitari D, Barkhof F, Fazekas F, Chabriat $\mathrm{H}$, LADIS Study group. Location of lacunar infarcts correlates with cognition in a sample of non-disabled subjects with age-related white-matter changes: the LADIS study. J Neurol Neurosurg Psychiatry 2009; 80: 478-483.

28 Hardy J, Selkoe DJ. The amyloid hypothesis of Alzheimer's disease: progress and problems on the road to therapeutics. Science 2002; 297: 353-356.

29 Newman AB, Fitzpatrick AL, Lopez O, Jackson S, Lyketsos C, Jagust W, Ives D, Dekosky ST, Kuller LH. Dementia and Alzheimer's disease incidence in relationship to cardiovascular disease in the Cardiovascular Health Study cohort. J Am Geriatr Soc 2005; 53: 1101-1107

30 de la Torre JC. Alzheimer disease as a vascular disorder: nosological evidence. Stroke 2002; 33: 1152-1162.

31 Matsuda H. Role of neuroimaging in Alzheimer's disease, with emphasis on brain perfusion SPECT. J Nucl Med 2007; 48: 1289-1300.

32 Chetelat G, Desgranges B, de la Sayette V, Viader F, Eustache F, Baron JC. Mild cognitive impairment: Can FDG-PET predict who is to rapidly convert to Alzheimer's disease? Neurology 2003; 60: 1374-1377.

33 Wolfe N, Reed BR, Eberling JL, Jagust WJ. Temporal lobe perfusion on single photon emission computed tomography predicts the rate of cognitive decline in Alzheimer's Disease. Arch Neurol 1995; 52: 257-262.

34 Nagata K, Buchan RJ, Yokoyama E, Kondoh Y, Sato M, Terashi H, Satoh Y, Watahiki Y, Senova M, Hirata Y, Hatazawa J. Misery perfusion with preserved vascular reactivity in Alzheimer's disease. Ann NY Acad Sci 1997; 826: 272-281.

35 De Rango P, Caso V, Leys D, Paciaroni M, Lenti M, Cao P. The role of carotid artery stenting and carotid endarterectomy in cognitive performance. A systematic review. Stroke 2008; 39: 3116-3127.

36 Imaizumi M, Kitagawa K, Hashikawa K, Oku N, Teratani T, Takasawa M, Yoshikawa T, Rishu P, Ohtsuki T, Hori M, Matsumoto M, Nishimura T. Detection of misery perfusion with split-dose 123I-iodoamphetamine single-photon emission computed tomography in patients with carotid occlusive diseases. Stroke 2002; 33: 2217-2223.

37 Kuroda S, Shiga T, Ishikawa T, Houkin K, Narita T, Katoh C, Tamaki N, Iwasaki Y. Reduced blood flow and preserved vasoreactivity characterize oxygen hypometabolism due to incomplete infarction in occlusive carotid artery diseases. J Nucl Med 2004; 45: 943-949.

38 Meyer JS, Konno S, Margishvili GM, Terayama Y. Vasodilator responses to acetazolamide tested in subtypes of vascular dementia. J Stroke Cerebrovasc Dis 1998; 7: 323-329. 